\title{
Bullous Erythema Nodosum Leprosum Through the Dermoscope
}

\author{
Deepak Vashisht ${ }^{1}$, Shekhar Neema ${ }^{1}$, Durga Madhab Tripathy ${ }^{1}$, Prashant Sengupta ${ }^{2}$
}

1 Department of Dermatology, Armed Forces Medical College, Pune, India

2 Department of Pathology, Armed Forces Medical College, Pune, India

Key words: bullous ENL, dermoscopy

Citation: Vashsisht D, Neema S, Tripathy DM, Sengupta P. Bullous erythema nodosum leprosum through the dermoscope. Dermatol Pract Concept. 2022;12(1):e2022027. DOI: https://doi.org/10.5826/dpc.1201a27

Accepted: June 29, 2021; Published: January 2022

Copyright: $\odot 2022$ Vashisht D et al. This is an open-access article distributed under the terms of the Creative Commons Attribution License BY-NC-4.0, https://creativecommons.org/licenses/by-nc/4.0/, which permits unrestricted noncommercial use, distribution, and reproduction in any medium, provided the original authors and source are credited.

Funding: None.

Competing interests: None.

Authorship: All authors have contributed significantly to this publication

Corresponding author: Durga Madhab Tripathy, Senior Resident, Department of Dermatology, Armed Forces Medical College, Pune, India. E-mail:dmt5861@gmail.com

\section{Introduction}

Erythema nodosum leprosum (ENL) forms a part of type 2 leprosy reaction characterized by crops of tender evanescent erythematous nodules that appear on extensors accompanied by systemic symptoms like fever and arthritis. Bullous ENL is a rare variant of the type 2 reaction characterized by multiple vesicles and flaccid bullae at sites of classic ENL with severe systemic symptoms. Other atypical variants include necrotic, hemorrhagic, purpuric, Sweet syndrome-like and erythema multiforme-like ENL.

Nowadays, dermoscopy is routinely employed in the diagnosis of leprosy, and it shows features akin to granulomatous dermatoses, explicitly, yellow-orange background reminiscent of underlying granulomas and few specific features. Specific features in the tuberculoid pole include loss of appendages and in the lepromatous pole xerosis, scaling, and hypopigmentation. Leprosy reactions are characterized by vascular changes in the form of arborizing blood vessels in ENL and diffuse erythema in type 1 reaction [1].
We report dermoscopic findings of bullous ENL lesions in a patient, findings that revealed both typical and atypical features.

\section{Case Presentation}

A 37-year-old male, with a known case of Hansen disease (borderline lepromatous leprosy) and on multidrug therapy consisting of rifampicin $600 \mathrm{mg}$ monthly, dapsone $100 \mathrm{mg}$ and clofazimine $50 \mathrm{mg}$ daily for 1 year, presented with multiple red raised, painful, erythematous nodules distributed symmetrically over the face, back, and upper limbs. Vesicles and bullae containing clear fluid were superimposed on most lesions (Figure 1, A and B). There was associated redness of the eyes, a high-grade fever, and joint pains. Mucosal surfaces, palms and soles were not involved, and Nikolsky sign was negative.

Tzanck smear showed neutrophils, and acantholytic cells were absent. A slit-skin smear test for acid-fast bacilli (Mycobacterium leprae) from 8 different sites, including 


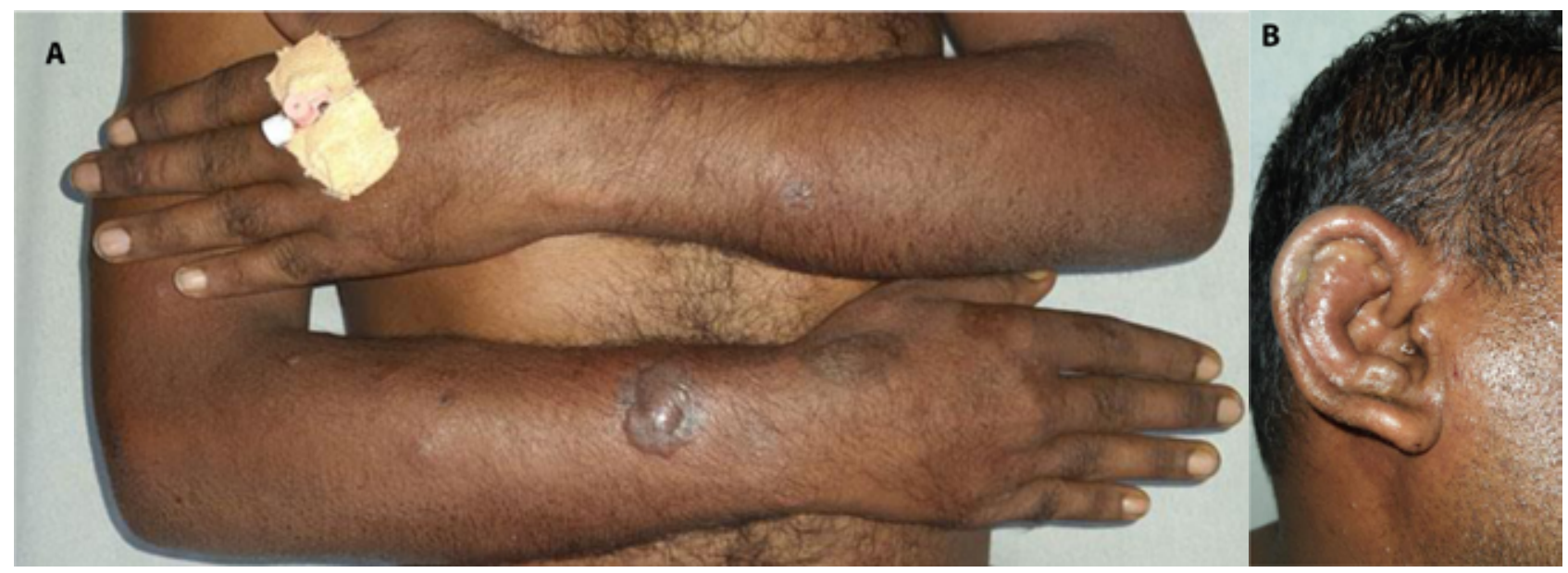

Figure 1. (A) Two ruptured bullae containing clear fluid located on both the forearms. (B) Solitary larger ruptured bullae over the right pinna.

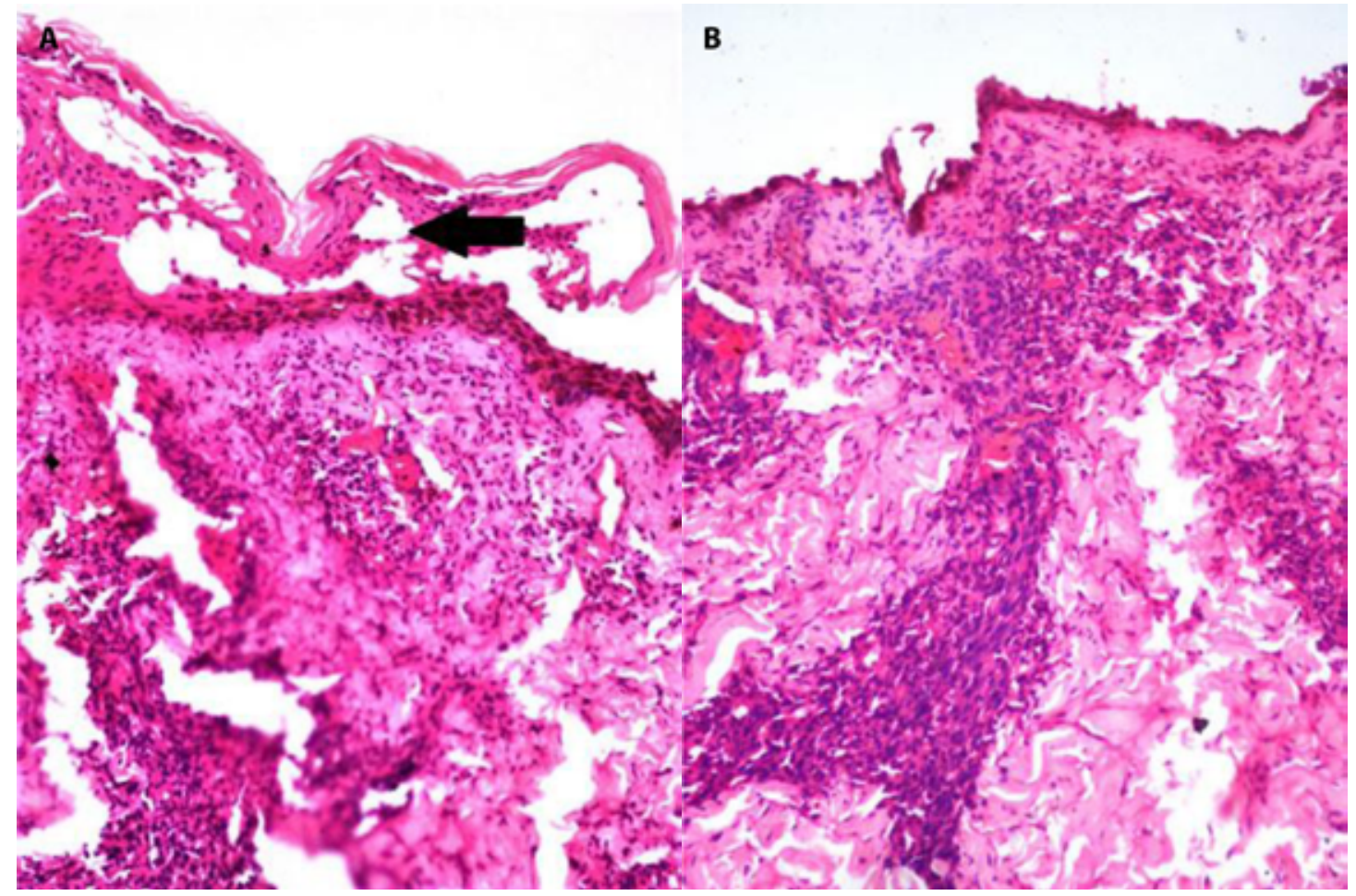

Figure 2. Histopathologic examination. (A) An intraepidermal split with spongiosis and neutrophils ( $\times 40$ ) (black arrow). (B) Multiple ill-formed granulomas comprised of epithelioid cells and lymphocytes, and foamy histiocytes were noted surrounding the dermal nerves and appendages.

lesions, showed an average bacteriological index of $4+$. Histopathology of the involved skin showed an intraepidermal separation, spongiosis, and neutrophilic infiltrate. Multiple ill-formed granulomas comprising of epithelioid cells, lymphocytes, and foamy histiocytes were noted surrounding the dermal nerves and appendages (Figure 2, A and B). Dermoscopy was performed using handheld DermLite DL4 dermatoscope, and images were captured with a Samsung phone. It showed a homogeneous white-pink area with an irregular border and surrounding erythema, similar to findings mentioned in the literature [2]. A closer view showed a few atypical and novel features in the form of a crumpled fabric appearance of white-pink areas and brown-gray dots and globules at the periphery (Figure 3, A and B).

The patient was managed as a type 2 leprosy reaction (bullous ENL) with prednisolone $40 \mathrm{mg} /$ day and thalidomide $100 \mathrm{mg} 4$ times a day. He responded to treatment and is currently on tapering doses of steroids and thalidomide

\section{Conclusions}

Leprosy has always eluded dermatologists with its varied presentations, and bullous ENL is an excellent example. With the growing popularity of dermoscopy in the diagnosis of 


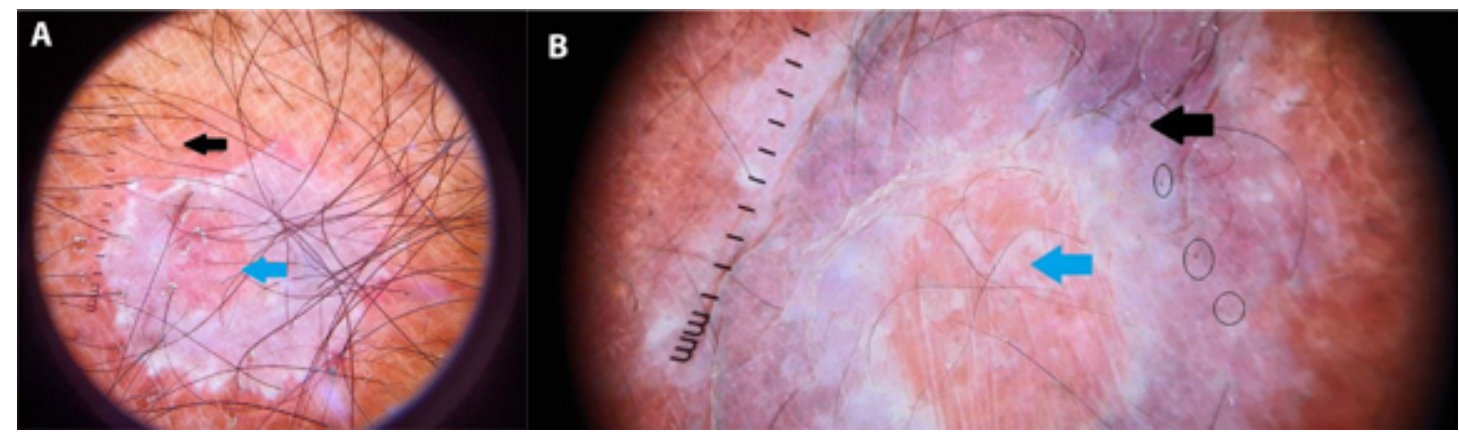

Figure 3. Dermoscopy. (A) A homogeneous white-pink area with irregular borders (blue arrow) and surrounding erythema (black arrow). (B) A closer view showed a few atypical and novel features in the form of a crumpled fabric appearance of white-pink areas (blue arrow) and brown-gray dots and globules at the periphery (black arrow and circles).

leprosy, we tried to analyze dermoscopic findings of bullous ENL lesions. The novelty of this case lies in intriguing clinical aspects and newer perspectives through the dermoscope.

Informed consent: Written informed consent for publication of clinical details and clinical images was obtained from the patient.

\section{References}

1. Vinay K, Kamat D, Chatterjee D, Narang T, Dogra S. Dermatoscopy in leprosy and its correlation with clinical spectrum and histopathology: a prospective observational study. J Eur Acad Dermatol Venereol. 2019;33(10):1947-1951. DOI: 10.1111/ jdv.15635. PMID: 31004456.

2. Chopra A, Mitra D, Agarwal R, Saraswat N, Talukdar K, Solanki A. Correlation of Dermoscopic and Histopathologic Patterns in Leprosy - A Pilot Study. Indian Dermatol Online J. 2019;10(6):663-668. DOI: 10.4103/idoj.IDOJ_297_18. PMID: 31807445. PMCID: PMC6859759. 